\title{
Circuit
}

Musiques contemporaines

\section{Le chant des ondes : sur la piste de Maurice Martenot, documentaire de Caroline Martel, Artifact/ONF, 2012}

\section{Maxime McKinley}

Volume 23, numéro 1, 2013

URI : https://id.erudit.org/iderudit/1017215ar

DOI : https://doi.org/10.7202/1017215ar

Aller au sommaire du numéro

Éditeur(s)

Les Presses de l’Université de Montréal

ISSN

1183-1693 (imprimé)

1488-9692 (numérique)

Découvrir la revue

Citer ce compte rendu

McKinley, M. (2013). Compte rendu de [Le chant des ondes : sur la piste de Maurice Martenot, documentaire de Caroline Martel, Artifact/ONF, 2012]. Circuit, 23(1), 79-81. https://doi.org/10.7202/1017215ar

Ce document est protégé par la loi sur le droit d'auteur. L'utilisation des services d'Érudit (y compris la reproduction) est assujettie à sa politique d'utilisation que vous pouvez consulter en ligne.

https://apropos.erudit.org/fr/usagers/politique-dutilisation/
Cet article est diffusé et préservé par Érudit.

Érudit est un consortium interuniversitaire sans but lucratif composé de l’Université de Montréal, l'Université Laval et l'Université du Québec à Montréal. Il a pour mission la promotion et la valorisation de la recherche. https://www.erudit.org/fr/ 


\title{
Le chant des ondes: sur la piste de Maurice Martenot,
}

\section{documentaire de Caroline Martel}

\author{
Artifact/ONF, 2012 \\ Compte rendu de Maxime McKinley
}

Le XXe siècle a été fécond en utopies et trouvailles porteuses de promesses pour le futur. Depuis quelques années, ce goût pour l'avenir est devenu un sujet d'étude pour les historiens, non sans un certain effet de rétro-futurisme. C'est à cette enseigne de la jeune histoire que loge le film Le chant des ondes, réalisé par Caroline Martel, et qui a été présenté en première mondiale aux Rencontres internationales du Documentaire de Montréal (RIDM, novembre 2012) ${ }^{1}$. Fort d'un remarquable travail d'archives, ce documentaire substantiel (97 minutes) retrace l'histoire des ondes Martenot, des toutes premières expérimentations sur les postes à lampes à triodes durant la Grande Guerre aux mégasconcerts du groupe Radiohead d'aujourd'hui.

Tel que nous le raconte le spécialiste Michel Risse, c'est par le truchement de la télégraphie sans fil (TSF) que le caporal Martenot, radiotélégraphiste durant la guerre de 1914-1918, découvrit le potentiel musical des ondes. L'invention et la sophistication de son instrument ne concernèrent donc pas l'émission du son - les ondes -, mais bien le contrôle de celui-ci, qu'il souhaitait aussi sensible et expressif que possible, à l'instar du violoncelle dont il jouait et dont on retrouve effectivement certaines inflexions idiomatiques au
Martenot (vibrato, portamento). En ce sens, il s'agissait d'expérimentations similaires - mais aux résultats différents - à celles de Léon Thérémine, dont le film montre d'ailleurs une archive de 1930.

C’est en 1928 que les ondes Martenot furent présentées pour la première fois publiquement, et le nouvel instrument eut un succès planétaire immédiat. Quelques années plus tard, en 1937, un disque promotionnel conçu pour l'Exposition des arts et techniques de Paris affirmait même avec enthousiasme: «Bientôt, il y aura dans chaque maison... un Martenot! » Le Martenot fut rapidement utilisé par des compositeurs, dont Arthur Honegger dans des films aux esthétiques surréalistes et fantastiques tels que $\mathrm{La}$ fin du monde (1931) d'Abel Gance, L'idée (1932) de Berthold Bartosch ou encore La nuit fantastique (1941) de Marcel L'Herbier². Ici, dans un des plus beaux moments du film, la réalisatrice, sans doute ravie de se trouver à la croisée de son art et de son sujet, multiplie les extraits cinématographiques, plus fascinants les uns que les autres.

Ce rapport au fantastique et à la science-fiction touche à un des aspects les plus prégnants du documentaire: une certaine mystique des ondes Martenot. 


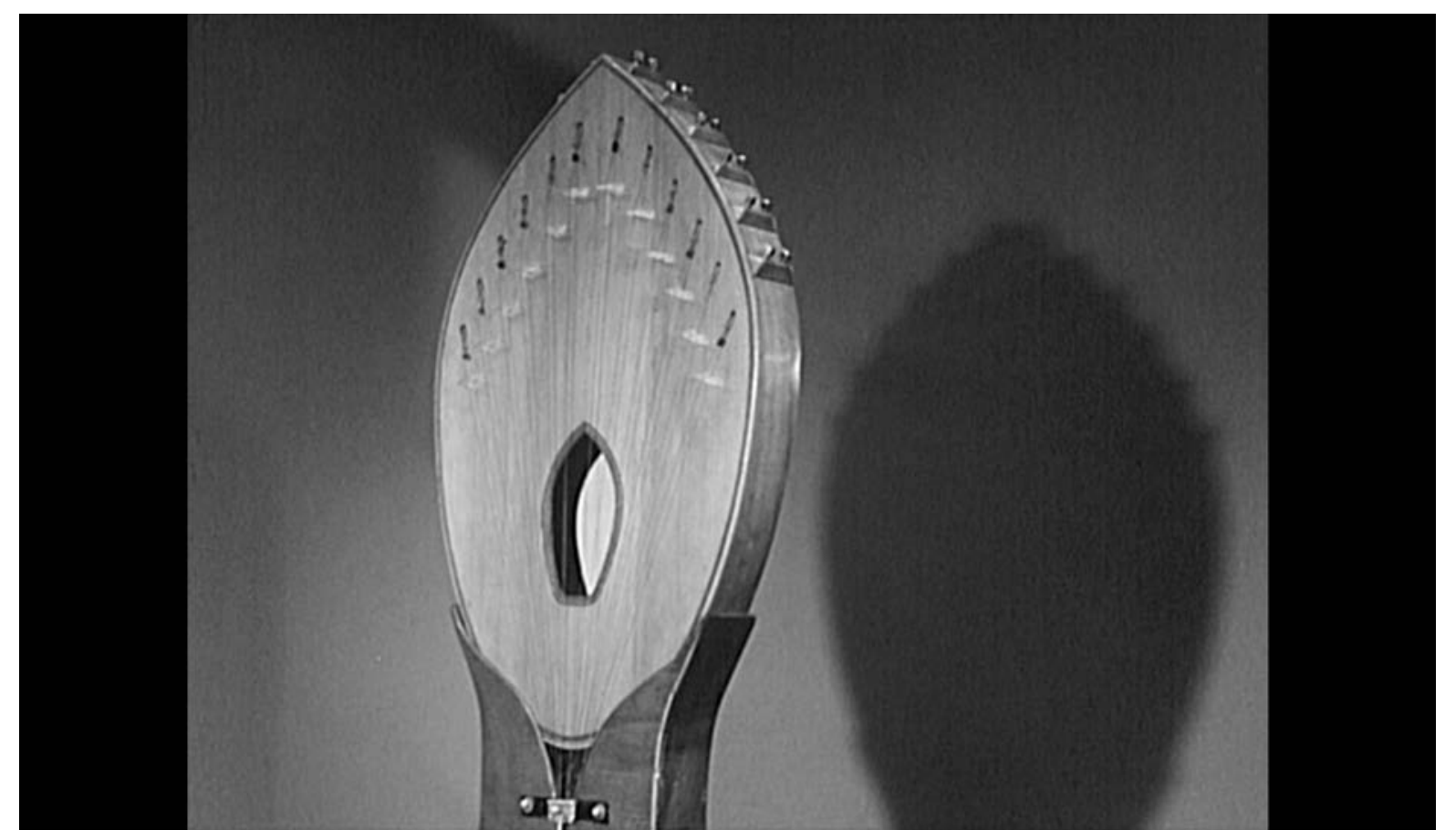

рното Image tirée du Chant des ondes de Caroline Martel (source: onf)

Certaines pages de christologie du philosophe Slavoj Žižek peuvent même s'avérer utiles pour décrire cette mystique 3 . En effet, Žižek décrit le Christ comme un «médiateur évanouissant» entre l'Au-delà et l'ici-bas, sa fragilité étant une condition même de cette médiation, puisqu'il lui faut disparaître pour que se répande l'Esprit saint sur les Fidèles. Tout au long du film, des commentaires établissent cette quasi-mystique du Martenot en tant que «médiateur évanouissant» venu de loin, mais dont la fragilité est inhérente à l'incarnation d'une dimension humaine, d'un influx vital, qui est aussi sa force. Cette fragilité humaine et vivante, contre la mécanique robotique, est d'ailleurs l'argument principal de ceux qui ne pensent pas que le Martenot soit rendu caduc par les fulgurants développements technologiques des plus récentes décennies. voire de l'imperfection -, Maurice Martenot répétait souvent ce principe, cité dans le film : «l'essentiel de la musique se passe à travers les impondérables».

Le Martenot comme «médiateur évanouissant» est un parallèle encore plus convaincant quand on sait jusqu'où allait l'imaginaire de son inventeur, c'est-àdire au rêve d'appliquer des branchements sur le cerveau de manière à ce que le chant des ondes puisse émerger directement de la pensée. Les extraits sonores exprimant le mieux cette dimension mystique liée au Martenot sont évidemment ceux de Messiaen, de La fête des belles eaux (1937) et de Saint François d'Assise (1983). Cette fragilité intrinsèque à l'instrument donnera lieu, dans le film, à beaucoup de passages beaucoup plus pragmatiques et techniques, notamment avec Jean-Loup Dierstein et Jean-Louis Martenot (fils de l'inventeur), quant à la survie - au sens matériel du 
terme - du Martenot, dont environ 70 instruments seulement seraient utilisés dans le monde actuellement.

C'est dire qu'il ne s'agit pas, dans cette histoire, que d'élévation cosmique. Au contraire, Maurice Martenot, dont un pan important de la vie fut voué à la pédagogie, souhaitait que son instrument s'implante dans la vie quotidienne des masses. Ainsi, il est question, tout au long du film, du Martenot dans des contextes relevant de la culture populaire: du swing à Édith Piaf ( Je t'ai dans la peau»), en passant par Harmonium et Beau Dommage. À ce titre, une rencontre passionnante - et un moment fort du documentaire - est celle de l'ondiste Suzanne Binet-Audet avec le guitariste, compositeur et multi-instrumentiste Jonny Greenwood, du groupe Radiohead, qui utilise volontiers les ondes Martenot. Greenwood compare cette rencontre à ce qu'aurait pu être celle d'Elton John et de Glenn Gould, rapprochement qui s'applique à la fois sur les plans artistique (populaire/savant) et géographique (Angleterre/Canada). Une autre comparaison de Greenwood est celle qu'il fait entre le Martenot et le saxophone: tous deux portent le nom de leurs inventeurs, mais ils ont surtout en commun d'avoir infiltré d'emblée plusieurs sphères culturelles, savantes et populaires. Pourtant, Greenwood évoque l'hypothèse, triste selon lui, que nous soyons passés «à côté » du Martenot, notamment à cause de ses caractéristiques lyriques apparues en pleine période d'anti-lyrisme, du moins parmi une certaine avant-garde.

Cette évocation de la précarité de l'héritage du Martenot touche à une corde sensible que partagent bien des sujets de la jeune histoire musicale. S'agit-il d'un enthousiasme éphémère, d'une utopie de passage destinée à ne subsister qu'à titre de simple curiosité historique, ou alors le Martenot n'est-il qu'au début de son histoire, la précarité de sa situation actuelle correspondant à la traversée d'un certain purgatoire, avant son implantation profonde et définitive dans la civilisation? Quoi qu'il en soit, dans ce film, la force de plusieurs des œuvres qu'on y entend demeure le plus puissant avocat pour plaider en faveur de la survivance de cet instrument ${ }^{4}$.

1. Le film poursuit sa route depuis. Il a notamment été sélectionné au $42^{\mathrm{e}}$ Festival international du film de Rotterdam (janvier 2013), et présenté au Cinéma Excentris de Montréal (du 15 mars au 11 avril 2013).

2. À noter qu'il y a au sujet de la musique de La nuit fantastique une contradiction entre ce qu'indiquent le documentaire et son générique : durant le visionnement, on apprend que la musique est de Marcel Delannoy, mais dans le générique, qu'elle est de Maurice Thiriet. D’après les premières entrées Internet sur le titre du film, il semble que ce soit le générique qui ait raison. L'année de parution du film, quant à elle, oscille entre 1941 et 1942, selon les sources. On notera, cependant, que l'Internet Movie Database donne la date de 1942 ainsi que le nom de Maurice Thiriet pour la musique. Voir:

$<$ www.imdb.com/title/tto041705/fullcredits?ref_=tt_ov_st_sm\#cast> (consulté le 5 février 2013).

3. Voir, par exemple, Slavoj Žižek ([2000]2010), Fragile absolu : pourquoi l'héritage chrétien vaut-il d'être défendu?, trad. de François Théron, Paris, Flammarion.

4. Il faut ici rendre hommage au travail remarquable fait au Québec par le pionnier Jean Laurendeau, que ce soit à titre de soliste - dans des œuvres marquantes comme Le cheminement de la baleine de Michel Gonneville -, ou au sein de l'Ensemble d'ondes de Montréal, à l'origine de beaucoup d'œuvres à (re)découvrir - pensons aux Jardins suspendus de Serge Provost, enregistrés en 1992 (SNE-574-CD) -, sans mentionner ses activités de pédagogue et d'auteur. 